\title{
Steady-State, Nonlinear Analysis of Large Arrays of Electrically Actuated Micromembranes Vibrating in a Fluid
}

\author{
Alexandre Halbach · Christophe Geuzaine
}

the date of receipt and acceptance should be inserted later

\begin{abstract}
This paper describes a robust and efficient method to obtain the steady-state, nonlinear behaviour of large arrays of electrically actuated micromembranes vibrating in a fluid. The nonlinear electromechanical behavior and the multiple vibration harmonics it creates are fully taken into account thanks to a multiharmonic finite element formulation, generated automatically using symbolic calculation. A domain decomposition method allows to consider large arrays of micromembranes by efficiently distributing the computational cost on parallel computers. Two- and three- dimensional examples highlight the main properties of the proposed method.
\end{abstract}

Keywords Finite element analysis, electric elastic acoustic coupling, domain decomposition, nonlinear, multiphysics, CMUT

\section{Introduction}

Many applications require the computation of the nonlinear steady-state response to a time-harmonic excitation. Capacitive micromachined ultrasonic transducers (CMUTs), used in applications such as ultrasound imaging [1,2] and nondestructive inspection [3], are a typical example. CMUTs consist in large arrays of electrically actuated micromembranes vibrating in a fluid: in order to evaluate the crosstalk deteriorating their imaging performance one may excite a given membrane in the array using a harmonic electric potential and compute the perturbation induced on the other membranes in the array.

University of Liège, Department of Electrical Engineering and Computer Science,

Montefiore Institute B28, B-4000 Liège, Belgium

E-mail: alexandre.halbach@ulg.ac.be·cgeuzaine@ulg.ac.be
While simple lumped-parameter models exist to model CMUTs, an accurate simulation requires the solution of the coupled partial differential equations representing the coupled elastodynamic, electrostatic and acoustic system. This coupled problem is nonlinear, due to the dependency of the electrostatic force with respect to the displacement of the micromembranes. Moreover, the crosstalk in CMUTs depends on the position of the membrane in the array, and the problem can therefore not be accurately solved simply by supposing clamped-clamped membranes [4] or periodic boundary conditions [5]. In this paper we propose to use finite elements to solve the fully coupled nonlinear problem for large arrays of membranes, without any simplifying assumption like supposing flat CMUTs in order to use the Rayleigh integral to compute the pressure field [6], neglecting the fluid coupling between distant membranes [6] or considering a linearized behavior [6,7].

While previous attempts have focused on this nonlinear problem for a single electromechanic micromembrane vibrating in vacuum in steady-state [8,9] and for the transient simulation of arrays thereof [10], to the best of our knowledge no scalable approach has been proposed so far for efficiently modelling the steady behaviour of large arrays vibrating in a fluid. To this end, we propose to combine a steady-state resolution method fully taking into account the nonlinear behavior thanks to an automatic multi-harmonic high-order finite element formulation, coupled with a domain decomposition technique to take advantage of parallel processing.

The paper is organised as follows. In section 2 the multiharmonic finite element formulation is presented on a simple electrostatic example before being applied to the full nonlinear electroelastoacoustic problem. Section 3 presents the domain decomposition resolution scheme. Section 4 presents numerical results on models of 2D and large realistic 3D ar- 
rays of electrically actuated micromembranes vibrating in water.

\section{Multiharmonic Finite Element Formulation}

The steady-state solution of the considered nonlinear problem is classically obtained using a time-stepping method (e.g. Newmark's method [11,12]), iterated until the steadystate is reached. However, since CMUTs are excited close to the resonance frequency of the micromembranes, such timedomain approaches require an enormous amount of timesteps to reach steady-state. An alternative is a time-harmonic resolution (in Fourier space) to directly obtain the desired steady-state solution. Because of the nonlinearity however additional harmonics appear in addition to the single exitation frequency, and their coupling has to be taken into account. Truncating the Fourier series of the unknown fields and approximating the Fourier coefficients by finite elements in this case leads to a large coupled nonlinear system. This method is called the multiharmonic or harmonic balance finite element method [13, 14, 15]. This multiharmonic strategy has already been investigated to study the nonlinear vibration of electrically actuated micromembranes in vacuum [16, 8, 9], as well as in several other research fields [17, 18, 19, 13, 20,21]. Its effective use on large scale applications is however impeded by two main factors. On the one hand the derivation of the equation terms in the multiharmonic formulation (even if the number $N_{h}$ of considered harmonics is as small as 2) can become extremely tedious when done manually, in particular if the nonlinear system is to be solved using a Newton-Raphson scheme on a deforming mesh. On the other hand the size of the nonlinear system is multiplied by $N_{h}$ compared to the time-domain approach. (Note that the convergence of the Fourier approximation is generally of or$\operatorname{der} N_{h}^{-1}$ [22], but can be much faster for simple excitations [17,23]).

The first issue is adressed below by using a fully automatic implementation of the multiharmonic formulation using symbolic computation, as already described in [24]. To alleviate the second issue, a domain decomposition technique will be introduced in Section 3 to split the problem into smaller, computationally manageable pieces. In addition, it will be shown in Section 4 that in practice only a few number of harmonics have to be considered to get a very accurate solution.

\subsection{Simple Electrostatic Example}

Let us consider a simple electrostatic problem solved in terms of the electrostatic potential $v(\mathbf{x}, t)$ on a multiharmonically vibrating mesh, deformed by the mechanical displacement $\mathbf{u}(\mathbf{x}, t)$. The system is excited via a time-harmonic Dirichlet boundary condition on the electric potential on an electrode $\Gamma_{e}:\left.v\right|_{\Gamma_{e}}(t)=\left.V_{1}\right|_{\Gamma_{e}} \sin \left(2 \pi f_{0} t\right):=\bar{v}(t)$. A reference potential of 0 is imposed on the ground (see Fig. 2, top). Let us assume that the electric potential solution and the mechanical displacement can be approximated by the following truncated Fourier series:

$v(\mathbf{x}, t)=V_{1}(\mathbf{x}) \sin \left(2 \pi f_{0} t\right)+V_{3}(\mathbf{x}) \sin \left(3 \cdot 2 \pi f_{0} t\right)$,

$\mathbf{u}(\mathbf{x}, t)=\mathbf{U}_{0}(\mathbf{x})+\mathbf{U}_{2}(\mathbf{x}) \cos \left(2 \cdot 2 \pi f_{0} t\right)$,

i.e., considering the first and third harmonic for the electric potential and the constant term and second harmonic for the displacement. While this limited expansion is chosen for the simplicity of the following analytic calculations, it will be seen in Section 4 that it already leads in practice to very good numerical results. The goal of the multiharmonic resolution is to find the spatial fields $V_{1}(\mathbf{x}), V_{3}(\mathbf{x}), \mathbf{U}_{0}(\mathbf{x})$ and $\mathbf{U}_{2}(\mathbf{x})$, which are the coefficients in the Fourier expansion.

Since the mesh deformation $\mathbf{u}$ is decomposed as a sum of harmonics, integration on the mesh deformed by $\mathbf{u}$ must be handled carefully. In order to do so, all the quantities are brought back to an initial, undeformed mesh [25] by introducing the change of variables $\mathbf{x}^{*}=\mathbf{x}+\mathbf{u}$ with Jacobian $\mathbf{J}$. Denoting by $\Omega$ the undeformed configuration and by $\Omega^{*}$ the deformed one and using relations $d \Omega^{*}=|\mathbf{J}| d \Omega$ and $\nabla^{*}=\mathbf{J}^{-1} \nabla$ leads to the following weak formulation of the electrostatic problem, where $\varepsilon$ is the electric permittivity: Find $v(\mathbf{x})$ in an appropriate function space, with $v=\bar{v}$ on the electrode and 0 on the ground, such that

$-\int_{\Omega^{*}} \varepsilon\left(\nabla^{*} v\right)^{T} \nabla^{*} v^{\prime} d \Omega^{*}=0$

holds for all appropriate test functions $v^{\prime}(\mathbf{x})$. On the undeformed mesh the formulation becomes

$-\int_{\Omega} \varepsilon(\nabla v)^{T} \mathbf{J}^{-T} \mathbf{J}^{-1} \nabla v^{\prime}|\mathbf{J}| d \Omega=0$.

In a one-dimensional setting, one would have

$\mathbf{J}=\frac{\partial x^{*}}{\partial x}=\left[1+\frac{\partial u_{x}}{\partial x}\right], \quad \mathbf{J}^{-1}=\frac{\partial x}{\partial x^{*}}=\frac{1}{|\mathbf{J}|}$

and $|\mathbf{J}|=1+\frac{\partial u_{x}}{\partial x}$. Equation 4 would become:

$-\int_{\Omega} \varepsilon \frac{\partial v}{\partial x} \frac{\partial v^{\prime}}{\partial x} \frac{1}{|\mathbf{J}|} d \Omega=0$

In 2D and 3D extra Jacobian terms appear.

In order to obtain the multiharmonic formulation, the non-polynomial factor $\mathbf{G}:=\frac{1}{|\mathbf{J}|}$ is first computed in weak form: Find $\mathbf{G}$ such that

$\int_{\Omega} \mathbf{G}|\mathbf{J}| \mathbf{G}^{\prime} d \Omega=\int_{\Omega} \mathbf{G}^{\prime} d \Omega$

holds for appropriate test functions $\mathbf{G}^{\prime}$. In practice $\mathbf{G}$ is approximated in the same way as $\mathbf{u}$, i.e. in this case with two Fourier coefficients: $\mathbf{G}=\mathbf{G}_{\mathbf{0}}+\mathbf{G}_{\mathbf{2}} \cos \left(2 \cdot 2 \pi f_{0} t\right)$. 
The next step could then simply be to symbolically replace all quantities by their truncated Fourier expansion and then expand the whole formulation and multiply the sines and cosines together using recursively the following four identities to leave only sines and cosines of degree one but at higher frequencies:

$$
\begin{aligned}
& \cos (a) \cos (b)=\frac{\cos (a+b)}{2}+\frac{\cos (a-b)}{2} \\
& \sin (a) \sin (b)=\frac{\cos (a-b)}{2}-\frac{\cos (a+b)}{2} \\
& \sin (a) \cos (b)=\frac{\sin (a+b)}{2}+\frac{\sin (a-b)}{2} \\
& \cos (a) \sin (b)=\frac{\sin (a+b)}{2}-\frac{\sin (a-b)}{2}
\end{aligned}
$$

Doing so at this step would however make the number of expanded symbolic terms increase dramatically. In $1 \mathrm{D}$ it can be shown that it increases as $N^{2}$ because of the product between $G$ and $\frac{\partial v}{\partial x}$, while in 2D it increases as $N^{4}$. In order to avoid this issue and limit the number of terms one can precompute all products of terms that are known, i.e. all terms but the unknown and the test function. A discrete Fourier transform can be used to extract the most important harmonics. In practice we multiply all known terms 2 by 2 recursively until the whole term has been computed.

Then, we

- replace the multiplied known term and the unknown by their truncated Fourier series;

- expand the formulation (including applying any time derivative to the sines and cosines - cf. Section 2.37;

- use (7) to transform the sines and cosines powers and products into sums of higher frequency sines and cosines.

This amounts to transforming (5) into:

$$
\begin{aligned}
& -\int_{\Omega} \varepsilon \frac{\partial v}{\partial x} \frac{\partial v^{\prime}}{\partial x} \frac{1}{|J|} d \Omega=0 \\
\Leftrightarrow & -\int_{\Omega} \varepsilon \frac{\partial\left(V_{1} \sin \left(2 \pi f_{0} t\right)+V_{3} \sin \left(3 \cdot 2 \pi f_{0} t\right)\right)}{\partial x} \frac{\partial v^{\prime}}{\partial x} \\
& \cdot\left(G_{0}+G_{2} \cos \left(2 \cdot 2 \pi f_{0} t\right)\right) d \Omega=0 \\
\Leftrightarrow & -\int_{\Omega} \varepsilon\left[G_{0} \frac{\partial V_{1}}{\partial x} \sin \left(2 \pi f_{0} t\right)+G_{0} \frac{\partial V_{3}}{\partial x} \sin \left(3 \cdot 2 \pi f_{0} t\right)\right. \\
& +G_{2} \cos \left(2 \cdot 2 \pi f_{0} t\right) \frac{\partial V_{1}}{\partial x} \sin \left(2 \pi f_{0} t\right) \\
& \left.+G_{2} \cos \left(2 \cdot 2 \pi f_{0} t\right) \frac{\partial V_{3}}{\partial x} \sin \left(3 \cdot 2 \pi f_{0} t\right)\right] \frac{\partial v^{\prime}}{\partial x} d \Omega=0 \\
\Leftrightarrow & -\int_{\Omega} \varepsilon\left[\left(G_{0} \frac{\partial V_{1}}{\partial x}-\frac{1}{2} G_{2} \frac{\partial V_{1}}{\partial x}+\frac{1}{2} G_{2} \frac{\partial V_{3}}{\partial x}\right)\right. \\
& \cdot \sin \left(2 \pi f_{0} t\right)+\left(G_{0} \frac{\partial V_{3}}{\partial x}+\frac{1}{2} G_{2} \frac{\partial V_{1}}{\partial x}\right) \sin \left(3 \cdot 2 \pi f_{0} t\right) \\
& \left.+\left(\frac{1}{2} G_{2} \frac{\partial V_{3}}{\partial x}\right) \sin \left(5 \cdot 2 \pi f_{0} t\right)\right] \frac{\partial v^{\prime}}{\partial x} d \Omega=0,
\end{aligned}
$$

which is valid for any time $t$ and can thus be split into three independent equations, with the sine terms removed. Taking the equations corresponding to the Fourier expansion of $v$, i.e. the terms multipled by $\sin \left(2 \pi f_{0} t\right)$ and the ones multiplied by $\sin \left(3 \cdot 2 \pi f_{0} t\right)$ gives an excellent approximation of the actual electrostatic formulation and leads to the final multiharmonic formulation: Find $V_{1}$ and $V_{3}$ such that

$$
\left\{\begin{array}{l}
\int_{\Omega} \varepsilon\left(G_{0} \frac{\partial V_{1}}{\partial x}-\frac{1}{2} G_{2} \frac{\partial V_{1}}{\partial x}+\frac{1}{2} G_{2} \frac{\partial V_{3}}{\partial x}\right) \frac{\partial v^{\prime}}{\partial x} d \Omega=0 \\
\int_{\Omega} \varepsilon\left(G_{0} \frac{\partial V_{3}}{\partial x}+\frac{1}{2} G_{2} \frac{\partial V_{1}}{\partial x}\right) \frac{\partial v^{\prime}}{\partial x} d \Omega=0
\end{array}\right.
$$

holds for appropriate test functions $v^{\prime}$.

This system can be rewritten in matrix form as:

$$
\left[\begin{array}{ll}
\mathbf{K}_{V_{1} V_{1}} & \mathbf{K}_{V_{1} V_{3}} \\
\mathbf{K}_{V_{3} V_{1}} & \mathbf{K}_{V_{3} V_{3}}
\end{array}\right]\left[\begin{array}{l}
\mathbf{V}_{1} \\
\mathbf{V}_{3}
\end{array}\right]=\left[\begin{array}{c}
\mathbf{f}_{1} \\
0
\end{array}\right],
$$

where each of the four blocks can be generated using a classical (mono)harmonic finite element assembly procedure and where the right-hand-side incorporates the contribution of the nonhomogeneous Dirichlet boundary condition. In this paper the blocks are discretised using a high order hierarchical finite element method [26] (with polynomial orders ranging from 1 to 3 ) to get an accurate approximation of the solution.

As can be seen, even for a simple linear electrostatic problem the harmonics $V_{1}$ and $V_{3}$ can be coupled if the mesh is deformed. To understand this physically, simply consider a mechanical membrane vibrating harmonically as a sine wave, with a constant applied electrostatic voltage between two electrodes. Even though the electrostatic voltage on the electrode is constant the voltage inside the membrane will vary with time and thus the overall voltage will have a constant component plus a harmonic component. In case the membrane displacement is a constant or simply zero then the electric potential harmonics are uncoupled and the offdiagonal blocks $K_{V_{1} V_{3}}$ and $K_{V_{3} V_{1}}$ are zero as can be seen in (9) when $G_{2}$ is set to zero.

\subsection{Implementation Aspects}

It should be no surprise that the multiharmonic formulation for the $2 \mathrm{D}$ and in particular the 3D case will contain many terms, since the computation on the vibrating mesh is brought back via a change of coordinates back to the undeformed mesh. Nevertheless, all that is needed for the symbolic calculations is an algorithm able to perform symbolic expansions of polynomials into sums of monomials (e.g. $\left.2(a+b)^{2}=2 a^{2}+2 a b+2 b^{2}\right)$, readily available as expand in the Matlab software for example, and a function able to transform powered sines, cosines and products thereof into 
sums of non powered higher frequency harmonics (e.g. replace $\cos (x)^{2}$ by $\frac{1}{2}+\frac{1}{2} \cos (2 x)$ ) using recursively the four trigonometric identities in (7).

In practice the symbolic computation is a matter of seconds in 2D and minutes in 3D for the largest required stiffness matrices in our non-optimised Matlab test code, and it is thus negligeable compared to the overall simulation time.

\subsection{Nonlinear Electroelastoacoustic Formulation}

We can readily use the above multiharmonic framework to compute the steady-state behavior of 2D and 3D electroelastoacoustic systems. In order to avoid lengthy derivations only the 2D formulation is presented in what follows, in its time-dependent form. This form is what the multiharmonic method is fed with so as to automatically derive the corresponding multiharmonic formulation for a required number of harmonics.

Consider an electroelastoacoustic system with a mechanical subdomain $\Omega_{m}$, an acoustic (fluid) subdomain $\Omega_{f}$ and an electric subdomain $\Omega_{e}$. (A star superscript will here again denote a deformation by the displacement field.) Let $v$ [V] be the electrostatic potential defined on $\Omega=\Omega_{e}, \mathbf{u}$ [m] the displacement field defined on $\Omega_{m} \subset \Omega$ with components $u_{x}$ and $u_{y}, p[\mathrm{~Pa}]$ the acoustic pressure field defined in the fluid domain $\Omega_{f} \subset \Omega$ and $\mathcal{M}$ the $2 \mathrm{D}$ elasticity operator defined by $\mathcal{M}(\mathbf{u})=\left[\frac{\partial u_{x}}{\partial x} \frac{\partial u_{y}}{\partial y} \frac{\partial u_{x}}{\partial y}+\frac{\partial u_{y}}{\partial x}\right]^{T}$. We consider the following weak formulation of the electrostatic problem with Dirichlet boundary conditions on $\Gamma_{e} \subset \partial \Omega_{e}$ : Find $v$ with $v=\bar{v}$ on $\Gamma_{e}$ such that

$-\int_{\Omega_{e}^{*}} \varepsilon(\nabla v)^{T} \nabla v^{\prime} d \Omega^{*}=0$

holds for appropriate test functions $v^{\prime}$. Defining $E_{x}=\frac{\partial v}{\partial x}$ and $E_{y}=\frac{\partial v}{\partial y}$ and using the Frobenius matrix product $A: B=$ $\sum_{i, j} A_{i, j} B_{i, j}$, we consider the following weak formulation of the 2D linear elasticity problem with Dirichlet boundary conditions on $\Gamma_{m} \subset \partial \Omega_{m}$ : Find $\mathbf{u}$ with $\mathbf{u}=\overline{\mathbf{u}}$ on $\Gamma_{m}$ such that

$$
\begin{gathered}
\int_{\Omega_{m}} \frac{E}{1-v^{2}}\left[\begin{array}{ccc}
1 & v & 0 \\
v & 1 & 0 \\
0 & 0 & \frac{1-v}{2}
\end{array}\right] \mathcal{M}(\mathbf{u})^{T} \mathcal{M}\left(\mathbf{u}^{\prime}\right) d \Omega \\
+\int_{\Omega^{*}} \frac{\varepsilon}{2}\left[\begin{array}{cc}
E_{x}^{2}-E_{y}^{2} & 2 E_{x} E_{y} \\
2 E_{x} E_{y} & E_{y}^{2}-E_{x}^{2}
\end{array}\right]:\left[\nabla u_{x}^{\prime} \nabla u_{y}^{\prime}\right] d \Omega^{*} \\
+\int_{\Omega_{m} \cap \Omega_{f}} a p \mathbf{n}^{T} \mathbf{u}^{\prime} d \Omega-\int_{\Omega_{m}} \rho \frac{\partial^{2} \mathbf{u}^{T}}{\partial t^{2}} \mathbf{u}^{\prime} d \Omega=0
\end{gathered}
$$

holds for appropriate test functions $\mathbf{u}^{\prime}$, where $v$ is Poisson's ratio, $E$ Young's modulus and $\rho$ the mass density (all homogeneous anisotropic). The second term in (12) is the (nonlinear in $v$ ) electrostatic force computed using the virtual work principle, see e.g. [27]. The third term is the pressure force applied by the fluid on the fluid-mechanic interface; $a$ is a scalar constant improving the matrix conditionning as described below.

The wave equation is used to get the scalar pressure field in the air: Find the pressure $p$ in an appropriate function space such that

$$
\begin{gathered}
\int_{\Omega_{f}}(\nabla p)^{T} \nabla p^{\prime} d \Omega+\int_{\Omega_{f}} \frac{1}{c^{2}} \frac{d^{2} p}{d t^{2}} p^{\prime} d \Omega \\
-\int_{\Omega_{m} \cap \Omega_{f}} \frac{1}{a} \rho \mathbf{n}^{T} \frac{d^{2} \mathbf{u}}{d t^{2}} p^{\prime} d \Omega+\int_{\Gamma_{\infty}} \frac{1}{c} \frac{d p}{d t} p^{\prime} d \Gamma=0
\end{gathered}
$$

where $\rho$ and $c$ are the density and the sound speed in the fluid. The third term in (13) is Newton's equation linking the mechanical acceleration to the pressure gradient [28] while the last term is Sommerfeld's radiation condition on the fictitious boundary $\Gamma_{\infty}$ truncating the computational domain.

We consider a staggered coupling that consists in solving the linear electrostatic and elastoacoustic formulations (11)-(12 13) in alternance. After finite element discretisation the electrostatic formulation (11) leads to the linear system $\mathbf{K}_{\mathbf{v}} \mathbf{x}_{\mathbf{v}}=\mathbf{f}_{\mathbf{v}}$, whose multi-block form is illustrated in (10). If each harmonic $V_{i}(\mathbf{x})$ is expanded in terms of $N$ finite element basis functions $s_{j}(\mathbf{x}), j=1, \ldots, N$, as $V_{i}(\mathbf{x})=\sum_{j=1}^{N} V_{i j} s_{j}(\mathbf{x})$, the solution vector $\mathbf{x}_{\mathbf{v}}$ is of the form $\left[V_{11}, \ldots, V_{1 N}, V_{21}, \ldots, V_{2 N}, \ldots\right]$. The elastoacoustic formulation is discretised in a similar way, leading to the algebraic system $\mathbf{K}_{\mathbf{u}, \mathbf{p}} \mathbf{x}_{\mathbf{u}, \mathbf{p}}=\mathbf{f}_{\mathbf{u}, \mathbf{p}}$ for the unknown displacement and pressure vector $\mathbf{x}_{\mathbf{u}, \mathbf{p}}$.

Although heavier to solve, a fully coupled monolithic resolution based on [29] is also considered in our multiharmonic framework [30] for faster nonlinear convergence. In this case the nonlinear algebraic system $\mathbf{K}_{\mathbf{u}, \mathbf{p}, \mathbf{v}}(\mathbf{x}) \mathbf{x}_{\mathbf{u}, \mathbf{p}, \mathbf{v}}$ $=\mathbf{f}_{\mathbf{u}, \mathbf{p}, \mathbf{v}}(\mathbf{x})$ is solved for the unknown displacement, pressure and electric potential vector $\mathbf{x}_{\mathbf{u}, \mathbf{p}, \mathbf{v}}$ with a Newton iteration which conists in starting with an initial guess $\mathbf{x}_{\mathbf{0}}$ for $\mathbf{x}_{\mathbf{u}, \mathbf{p}, \mathbf{v}}$ and updating the guess at the $k t h$ iteration as follows:

$$
\begin{aligned}
\mathbf{J}\left(\mathbf{x}_{\mathbf{k}}\right) \mathbf{d x} & =\mathbf{b}\left(\mathbf{x}_{\mathbf{k}}\right)-\mathbf{K}\left(\mathbf{x}_{\mathbf{k}}\right) \mathbf{x}_{\mathbf{k}} \\
\mathbf{x}_{\mathbf{k}+\mathbf{1}} & =\mathbf{x}_{\mathbf{k}}+\mathbf{d x}
\end{aligned}
$$

where $\mathbf{J}$ is Newton's Jacobian matrix containing $\mathbf{K}$ plus the linearised force terms described in [29].

\section{Domain Decomposition Algorithm}

The fundamental idea of a domain decomposition method (DDM) is to split the overall domain into several overlapping or non-overlapping subdomains $\Omega_{i}, i=1, \ldots, N_{d}$ (Fig. 1) and solve iteratively the global problem, using local solutions on each subdomain computed in parallel. Several linear [31,32, 33, 34, 35] and nonlinear [36, 37, 38] domain decomposition algorithms have been developped. A previous 


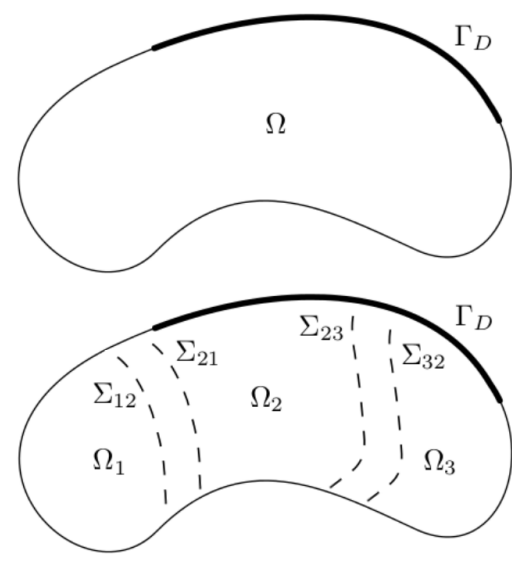

Fig. 1 Overlapping subdomains in the overall domain.

study [30] for nonlinear electroelastic problems compared some linear and nonlinear DD algorithms. Because the electroelastic nonlinearity is smooth in this paper an overlapping staggered [39] linear restricted additive Schwarz method [33] is considered.

At every nonlinear iteration the linear algebraic problems of the form $\mathbf{K x}=\mathbf{b}$ or $\mathbf{J d x}=\mathbf{b}-\mathbf{K x}$ (denoted $\mathbf{A x}=\mathbf{b}$ from now on) arising in the staggered and monolithic resolution methods described in the previous section are solved iteratively with a Krylov solver (e.g. with GMRES) in their preconditionned form:

$\mathbf{M}_{R A S}^{-1} \mathbf{A} \mathbf{x} \equiv \sum_{i=1}^{N_{d}} \tilde{\mathbf{R}}_{i}^{T} \mathbf{A}_{i}^{-1} \mathbf{R}_{i} \mathbf{A} \mathbf{x}=\sum_{i=1}^{N_{d}} \tilde{\mathbf{R}}_{i}^{T} \mathbf{A}_{i}^{-1} \mathbf{R}_{i} \mathbf{b} \equiv \mathbf{M}_{R A S}^{-1} \mathbf{b}$

with $\tilde{\mathbf{R}}_{i}$ and $\mathbf{R}_{i}$ the usual restriction operators respectively on the restricted, disjoint $\Omega_{i}$ subdomain and on $\Omega_{i}$. $\mathbf{R}_{i}$ is a matrix of zeros and ones with a number of rows equal to the number of algebraic unknowns in subdomain $\Omega_{i}$ and a number of columns equal to the number of degrees of freedom in the whole domain $\Omega$. Multiplying $\mathbf{R}_{i}$ by $\mathbf{x}$ gives the portion of $\mathbf{x}$ corresponding to $\Omega_{i}$. Multiplying $\tilde{\mathbf{R}}_{i}$ by $\mathbf{x}$ gives the portion of $\mathbf{x}$ corresponding to the 0-overlap disjoint restriction of $\Omega_{i}$. Each application of $\mathbf{A}_{i}^{-1}$ corresponds to the solution of the local problem on subdomain $i$, computed independently, in parallel. To the restricted additive Schwarz preconditionner $\mathbf{M}_{R A S}^{-1}$ a coarse grid preconditionner [33,40, 41] $\mathbf{R}_{\text {coarse }}^{T} \mathbf{A}_{\text {coarse }}^{-1} \mathbf{R}_{\text {coarse }}$ is added to make the domain decomposition convergence less dependent on the number of subdomains: $\mathbf{M}_{R A S}^{-1}:=\mathbf{M}_{R A S}^{-1}+\mathbf{R}_{\text {coarse }}^{T} \mathbf{A}_{\text {coarse }}^{-1} \mathbf{R}_{\text {coarse. }}$ In this paper both $\mathbf{A}^{-1}$ and the coarse grid resolution $\mathbf{A}_{\text {coarse }}^{-1}$ are performed on the same mesh: $\mathbf{A}^{-1}$ with high order (second or third) finite elements; $\mathbf{A}_{\text {coarse }}^{-1}$ with first order elements. Using hierarchical finite element bases [26] leads to trivial coarse-to-fine and fine-to-coarse interpolation matrices $\mathbf{R}_{\text {coarse }}$ and $\mathbf{R}_{\text {coarse }}^{T}$, consisting of only 0's and 1's.
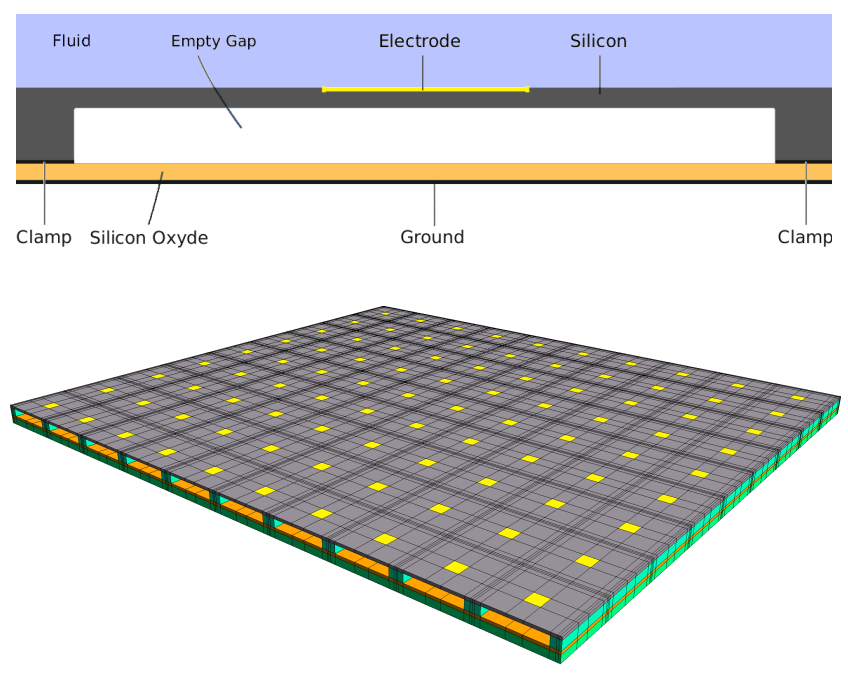

Fig. 2 Cut view of a single 3D membrane and perspective view of a 10 by 10 3D micromembrane array - fluid not displayed on the array. Not to scale.

\section{Numerical Results}

We consider both a 2D model of a $2 \times 1$ vibrating micromembrane array and a nxn 3D model: see Fig. 2. One cell consists of a silicon membrane vibrating in water due to the electrostatic force resulting from a sine voltage with a DC bias applied between the electrode and the ground. In the $3 \mathrm{D}$ case the electric ground is separated from the mechanical clamp by an insulating silicon dioxide layer. Water is considered for the fluid to better illustrate the crosstalk between cells, air leads to weaker crosstalk. Formulation (12) is solved in the silicon, formulation (11) is solved in the silicon, silicon dioxide and in the vacuum gap and formulation 13) is solved in the fluid. The whole mesh is made up of about 13,000 quadrangles in 2D (2 membranes) and 250,880 hexahedra in 3D (144 membranes and the outer fluid layer). Homogeneous Dirichlet boundary conditions are applied on the clamp for the elasticity formulation and on the ground for the electrostatic formulation.

The dimensions of the vibrating membranes are chosen close to the dimensions of a modern CMUT [42]. For the numerical tests, the following geometrical and material characteristics have been used: membrane length of $50 \mu \mathrm{m}$ (of which $40 \mu \mathrm{m}$ lie above the vacuum gap), solid support pillars thickness of $10 \mu \mathrm{m}$, membrane thickness $750 \mathrm{~nm}$, vacuum height $500 \mathrm{~nm}$ (pull-in expected for a displacement of about $\frac{1}{3} \cdot 500 \mathrm{~nm}$ ), electrode length of $10 \mu \mathrm{m}$; silicon and air with electric permittivity $\varepsilon_{\text {silicon }}=11.7 \cdot 8.854 \cdot 10^{-12}[\mathrm{~F} / \mathrm{m}]$, $\varepsilon_{\text {silicon dioxide }}=3.9 \cdot 8.854 \cdot 10^{-12}[\mathrm{~F} / \mathrm{m}]$ and $\varepsilon_{\text {vacuum }}=8.854$. $10^{-12}[\mathrm{~F} / \mathrm{m}]$, Young's modulus $E=150 \cdot 10^{9}\left[\mathrm{~N} / \mathrm{m}^{2}\right]$, Poisson's ratio $v=0.3$, density of water $\rho_{\text {water }}=1000\left[\mathrm{~kg} / \mathrm{m}^{3}\right]$, speed of sound in water $c_{\text {water }}=1484[\mathrm{~m} / \mathrm{s}]$. The water do- 
main in 2D is truncated after 4 membrane length $(200 \mu \mathrm{m})$ on the left and right side of the two membranes and after 10 membrane length $(500 \mu \mathrm{m})$ in the upper direction to limit the impact of the truncation to less than a few percent error on the maximum of the displacement harmonics. The constant part of the electric electrode-to-ground excitation is applied to all the membranes in the array (except in 3D on the exterior membranes used to move the fluid truncation further from the active membranes) while the alternating part of the excitation is only applied to a single one.

\subsection{Nonlinear Behavior}

In practical CMUT applications a close-to-linear vibration is achieved by adding a tiny alternating voltage (e.g. $1 \%$ of the pull-in voltage) to a big constant excitation voltage (e.g. $90 \%$ of the pull-in voltage). In this paper however the nonlinear behavior is put to the fore by applying a larger than usual alternating voltage. In the 2D test case an electrodeto-ground excitation of $v(t)=40+40 \sin (2 \pi \cdot 800000 t)$ [V] with a strong alternating voltage is chosen for a pull-in voltage of about $110 \mathrm{~V}$ and a first mode resonance at about $1 \mathrm{MHz}$. Let us denote the multiharmonic expansion of the displacement, pressure and electric potential field as

$\mathbf{u}(\mathbf{x}, t)=\mathbf{U}_{0}(\mathbf{x})+\mathbf{U}_{s 1}(\mathbf{x}) \sin \left(2 \pi f_{0} t\right)+\mathbf{U}_{c 1}(\mathbf{x}) \cos \left(2 \pi f_{0} t\right)+$ $\mathbf{U}_{s 2}(\mathbf{x}) \sin \left(4 \pi f_{0} t\right)+\mathbf{U}_{c 2}(\mathbf{x}) \cos \left(4 \pi f_{0} t\right)+\ldots, p(\mathbf{x}, t)=P_{0}(\mathbf{x})+$ $P_{s 1}(\mathbf{x}) \sin \left(2 \pi f_{0} t\right)+P_{c 1}(\mathbf{x}) \cos \left(2 \pi f_{0} t\right)+P_{s 2}(\mathbf{x}) \sin \left(4 \pi f_{0} t\right)+$ $P_{c 2}(\mathbf{x}) \cos \left(4 \pi f_{0} t\right)+\ldots$ and $v(\mathbf{x}, t)=V_{0}(\mathbf{x})+V_{s 1}(\mathbf{x}) \sin \left(2 \pi f_{0} t\right)+$ $V_{c 1}(\mathbf{x}) \cos \left(2 \pi f_{0} t\right)+V_{s 2}(\mathbf{x}) \sin \left(4 \pi f_{0} t\right)+V_{c 2}(\mathbf{x}) \cos \left(4 \pi f_{0} t\right)+$ $\ldots$ with $\mathbf{U}_{s 1}$ and $\mathbf{U}_{c 1}$ respectively the in-phase and quadrature vibration with respect to the electric sine excitation. The shape of the first 9 vibration harmonics (i.e. $\mathbf{U}_{0}, \mathbf{U}_{s 1}, \mathbf{U}_{c 1}$, $\mathbf{U}_{s 2}, \mathbf{U}_{c 2}, \mathbf{U}_{s 3}, \mathbf{U}_{c 3}, \mathbf{U}_{s 4}, \mathbf{U}_{c 4}$ for the displacement field) is displayed on Fig. 3 and the absolute magnitude of the displacement, the pressure and the electric potential in this test case is shown on Fig. 5. Fig. 4 shows the same simulation on three cross-talking membranes. Comparing Fig. 3 and Fig. 4 shows that the crosstalk and the array size dramatically influence the membrane vibration: as an example when a third unexcited membrane is added to the right of the two membranes the $\mathbf{U}_{s 1}$ vibration is significantly stronger.

The crosstalk and the nonlinear behavior are clearly visible on Fig. 3, where multiple mechanical vibration harmonics appear because of the nonlinearity. With a tiny alternating electric excitation component the Fourier terms $\mathbf{U}_{0}, \mathbf{U}_{s 1}$ and $\mathbf{U}_{c 1}$ are dominant: the behavior is close to linear. The terms $\mathbf{U}_{s 2}$ and $\mathbf{U}_{c 2}$ grow bigger as the alternating excitation component is increased and the constant component is decreased. If the constant component is dropped all what is left is $\mathbf{U}_{0}, \mathbf{U}_{s 2}, \mathbf{U}_{c 2}$ and higher harmonics. This makes physical sense since the electric force acting on the membrane will have the same direction no matter the sign of the alternating electric potential: the vibration frequency is doubled.

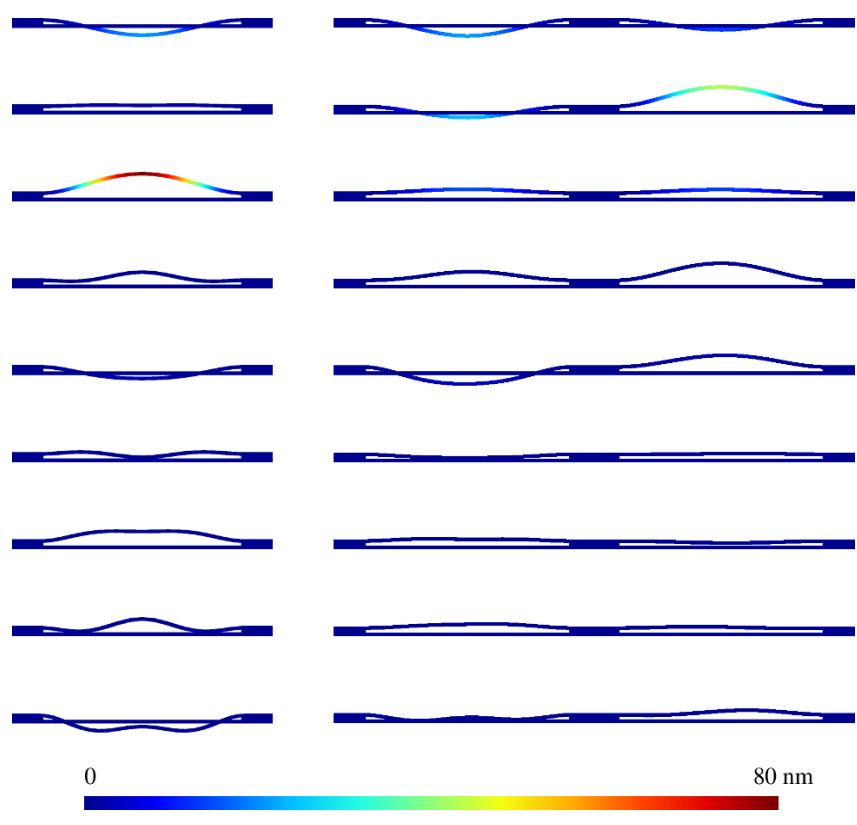

Fig. 3 Shape of the displacement harmonic $\mathbf{U}_{0}, \mathbf{U}_{s 1}, \mathbf{U}_{c 1}, \mathbf{U}_{s 2}, \mathbf{U}_{c 2}, \mathbf{U}_{s 3}$, $\mathbf{U}_{c 3}, \mathbf{U}_{s 4}, \mathbf{U}_{c 4}$ (from top to bottom) for a single (left) and two (right) membranes. In the latter case only the left membrane is dynamically excited. For a given harmonic the displacement is exagerated by the same factor for the left and right image.

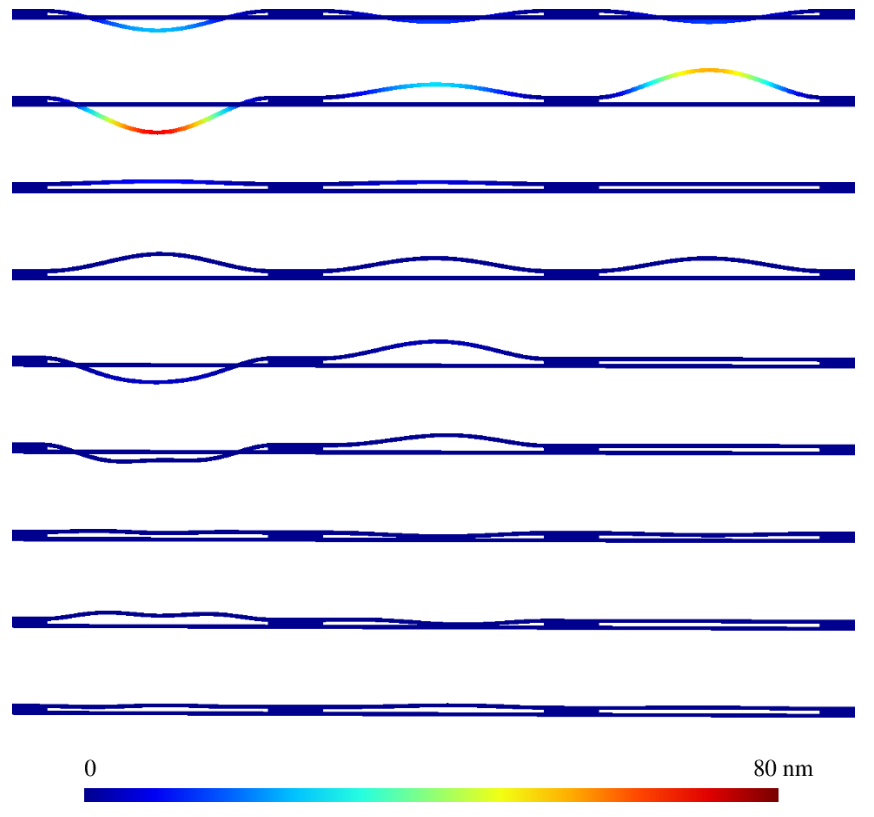

Fig. 4 Shape of the displacement harmonic $\mathbf{U}_{0}, \mathbf{U}_{s 1}, \mathbf{U}_{c 1}, \mathbf{U}_{s 2}, \mathbf{U}_{c 2}, \mathbf{U}_{s 3}$, $\mathbf{U}_{c 3}, \mathbf{U}_{s 4}, \mathbf{U}_{c 4}$ (from top to bottom) for three membranes. Displacements are exagerated by the same factor as in Fig. 3 


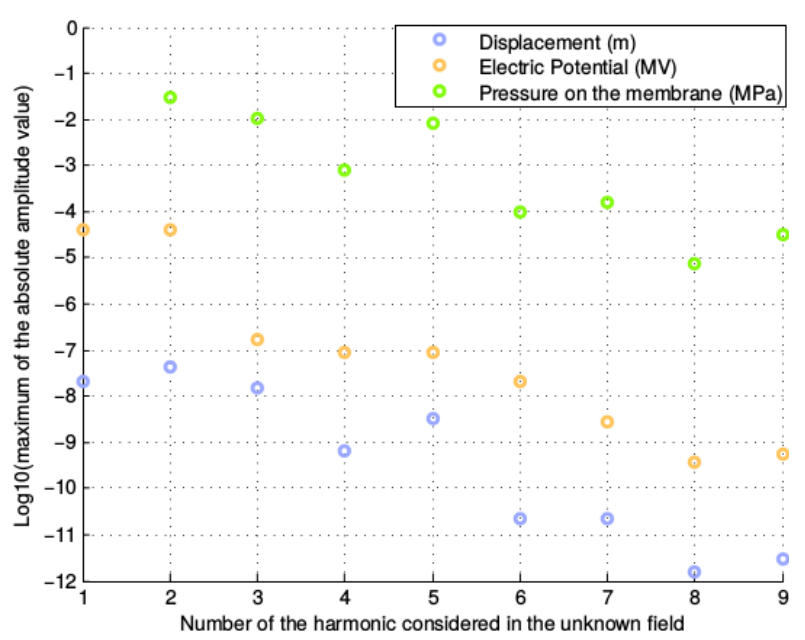

Fig. 5 Maximum absolute value displacement $(\mathrm{m})$, pressure (MPa, $10^{6} \cdot \mathrm{Pa}$ ) and electric potential $\left(\mathrm{MV}, 10^{6} \cdot \mathrm{V}\right.$ ) for each of the 9 first harmonics on the two membrane geometry. Pressure is measured at the membrane top.

Another impact of the nonlinear behavior is the appearance of new resonance peaks between $0 \mathrm{~Hz}$ and the first mode linear resonance frequency. As an example the Fourier term $\mathbf{U}_{c 2}$ vibrates at twice the $\mathbf{U}_{c 1}$ vibration frequency and resonates thus at an electric excitation frequency of about half the first mode linear resonance frequency. The bottom harmonics visible on Fig. 3 are clearly vibrating beyond their first resonance mode. An additional nonlinear behavior visible on the figure is the different time-constant deformation (top of the figure) on the left and right membranes, even though both have a same constant excitation of $40 \mathrm{~V}$. This comes from the coupling between harmonics when nonlinearity is considered.

\subsection{Multiharmonic Resolution}

Both the staggered and the monolithic (with Newton's method) resolutions are used in the multiharmonic framework to get the steady-state nonlinear solution of the electroelastoacoustic problem. Discretising this stiff structure-fluid interaction problem leads to an ill-conditionned algebraic problem [43]. Fortunately the pressure in the elastoacoustic and electroelastoacoustic coupled system can be scaled by the scalar factor $a$, as mentionned in Section $2.3\left(a=10^{10}\right.$ in this paper): the acoustic pressure is artificially divided by $a$ but to end up with the right solution the pressure force on the membrane is multiplied by $a$. Doing so brings the measured $10^{18}$ matrix conditionning in $3 \mathrm{D}$ back to $10^{6}$ (with an extra diagonal scaling). The pressure obviously needs to be multiplied by $a$ afterwards. Because the contribution to the nonlinear residual of the acoustic pressure is underestimated by a factor $a$ we multiply it by $a$.
As can be seen on Fig. 5 for an accurate solution only 5 harmonics need to be considered in the Fourier truncation of the pressure and displacement field while 2 are sufficient for the electric potential in the 2D test case settings. Solving for all harmonics at once in a multiharmonic resolution will lead to a much larger problem to solve than for a classical time resolution with e.g. a Newmark scheme. In the staggered resolution scheme of section 2.3 however, the large elastoacoustic problem 12 13 that needs to be solved at every nonlinear iteration of the multiharmonic resolution can be solved separately for every frequency, that is for $\mathbf{U}_{0}$ separately from the group $\mathbf{U}_{s 1}, \mathbf{U}_{c 1}, P_{s 1}$ and $P_{c 1}$, separately from $\mathbf{U}_{s 2}, \mathbf{U}_{c 2}, P_{s 2}$ and $P_{c 2} \ldots$ This decoupling results from the linearity of the elasticity and acoustic formulations and is illustrated in the sparsity pattern of Fig. 6 . For the smallersized electrostatic problem however all harmonics are coupled since the problem is computed on the displacementdeformed mesh.

Fig. 7 compares the three major time consuming steps in the multiharmonic resolution on the $2 \mathrm{D}$ test case with third order finite elements, for the electrostatic formulation (11), for the electrostatic force and for Newton's Jacobian when an increasing number of harmonics is considered in the Fourier truncation of the unknown fields. The time required to do all prior symbolic computation (done only once) is compared to the time required to generate the stiffness matrices in the multiharmonic framework and to compute their LU decomposition (timings for the 3D problems can be obtained in [24]). As expected Newton's Jacobian dominates all timings and should thus be used only when its faster nonlinear convergence makes it worthwhile. The symbolic computation time takes a non negligeable proportion of the generation and LU decomposition times. It can however be computed once and for all when starting the process and its cost is independent of the number of elements in the mesh. The generation time also takes longer than the actual LU decomposition time in this $2 \mathrm{D}$ setting. In any case for the staggered nonlinear resolution scheme where all harmonics need to be solved together only for the electrostatic problem a large number of harmonics can be treated in a reasonable amount of time both in 2D and in 3D [24]. Finally it is worth noting that the matrix conditionning for both the electrostatic formulation and Newton's Jacobian stay stable with an increasing number of harmonics (Fig. 8).

\subsection{Comparison with Time-Domain Solver}

In the $2 \mathrm{D}$ test case, when the electric excitation frequency is set closer to membrane resonance, a classical Newmark scheme [11,12] has been observed to bring the membrane beyond pull-in due to a transient displacement overshoot, never reaching steady-state. When steady-state can be reached 

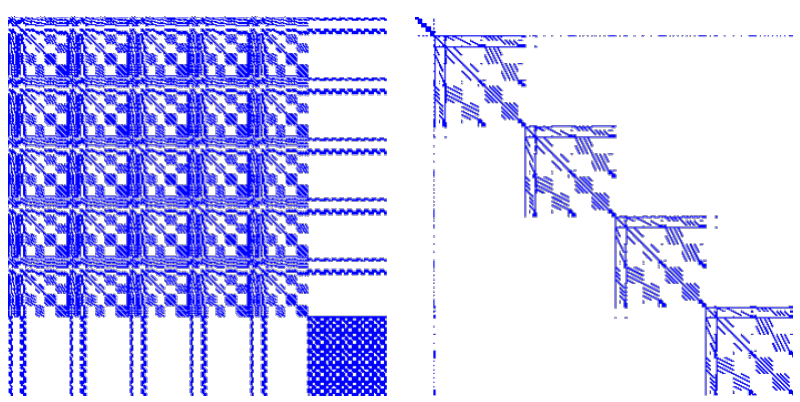

Fig. 6 Sparsity pattern of the electrostatic stiffness matrix (left) and elastoacoustic stiffness matrix (right) for 5 harmonics per unknown field.

(with an electric excitation of $800 \mathrm{kHz}$ ) the transient oscillations still need several tens of electric excitation periods to be decently damped (thanks to the emitted acoustic power, a major damping cause in water) as shown on Fig. 9 This figure compares for the first 5 displacement harmonics the maximum of the multiharmonically computed harmonics (horizontal lines) with the harmonics computed via a Fourier transform at every electric excitation period in the Newmark time resolution. With at least 200 timesteps per period there is a good match between both resolution methods but the larger size of the multiharmonic resolution is massively overcompensated by the large number of time steps needed to correctly approximate the steady-state in the time resolution. The multiharmonic resolution is in this case orders of magnitudes faster than the classical time resolution.

\subsection{Large 3D Array with Domain Decomposition}

Our multiharmonic framework combined to the domain decomposition method can be used to compute challenging nonlinear 3D problems. The steady-state solution was computed for a large 3D CMUT array vibrating in water. The array contains 144 elementary 3D cells whose cross-section is depicted on Fig. 2 For high accuracy seven harmonics have been used in the Fourier truncation of the displacement, pressure and electric potential fields: out of the 7 the last 2 are negligeable while the first and the fifth are the largest. After discretisation there are 2,206,526 degrees of freedom in the unknown vector $\mathbf{x}_{\mathbf{v}}$ of the electrostatic problem and $18,305,463$ in $\mathbf{x}_{\mathbf{u}, \mathbf{p}}$ for the elastoacoustic problem. The solution time on 196 processing units $(2 \mathrm{GHz}$ Intel cores with 4 GB RAM) was about 8 hours per staggered nonlinear iteration with a non-optimised Matlab implementation (3 iterations accurately solve the nonlinearity). Parallel to the membrane, structured mesh layers made up of $16 \times 16$ order 2 hexahedra were used. The fluid was 2 membrane-length $(100 \mu \mathrm{m})$ high and meshed with 2 layers. Fig. 11 shows the nonlinear solution for displacement and pressure harmonics $\mathbf{U}_{c 5}$ and $P_{c 5}$ in case of a 70 volts electrode to ground DC bias
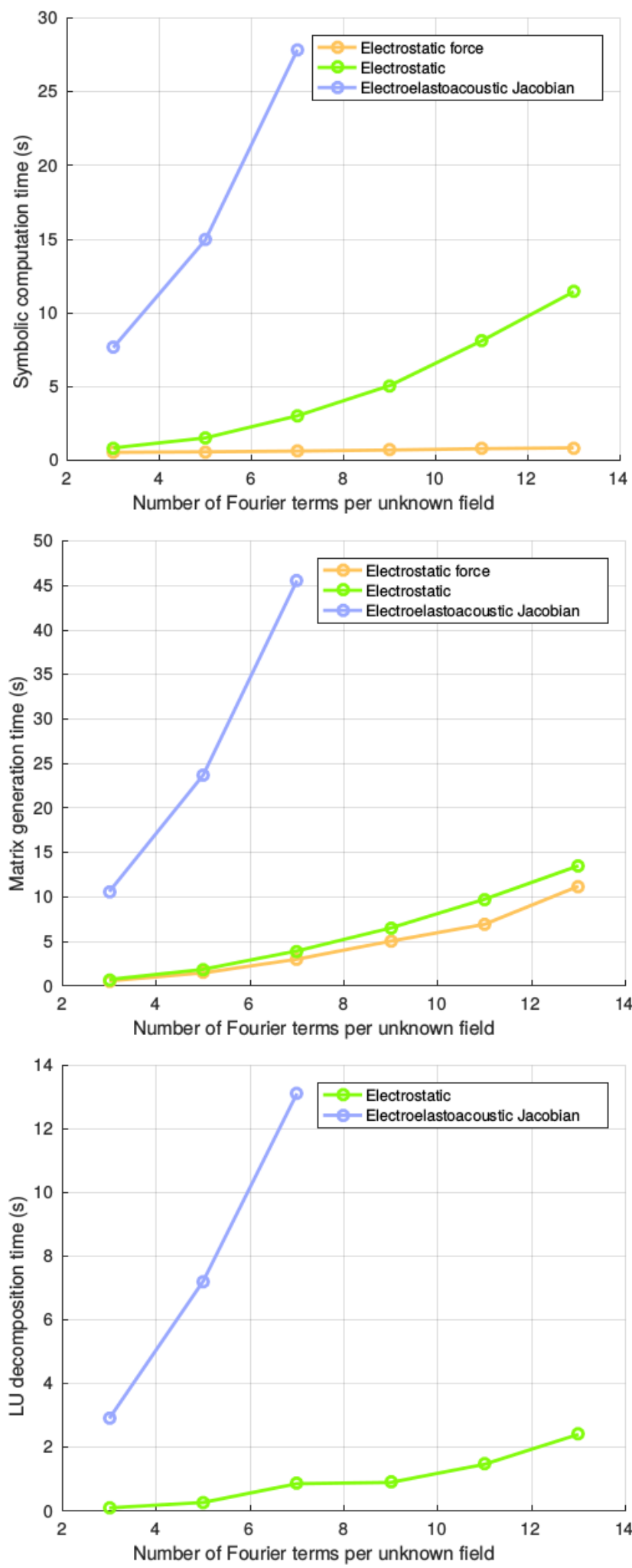

Fig. 7 Symbolic computation (top), matrix generation (middle) and LU decomposition (bottom) time (s) versus number of terms in each unknown field Fourier expansion for the 2D electrostatic 11 formulation, the electrostatic force and Newton's Jacobian. 


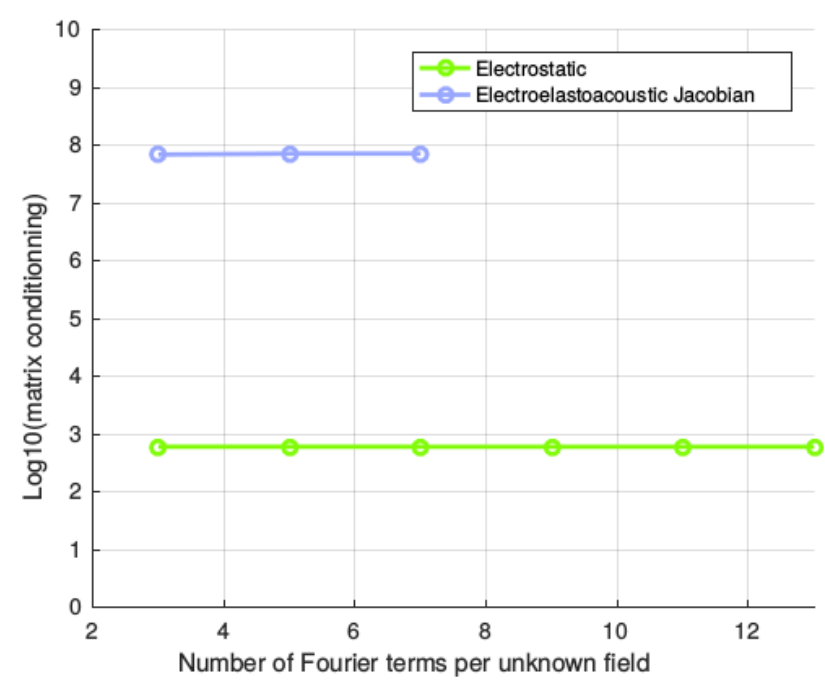

Fig. 8 Matrix conditionning (after diagonal scaling) versus number of terms in each unknown field Fourier expansion for the 2D electrostatic formulation 11 and Newton's Jacobian.
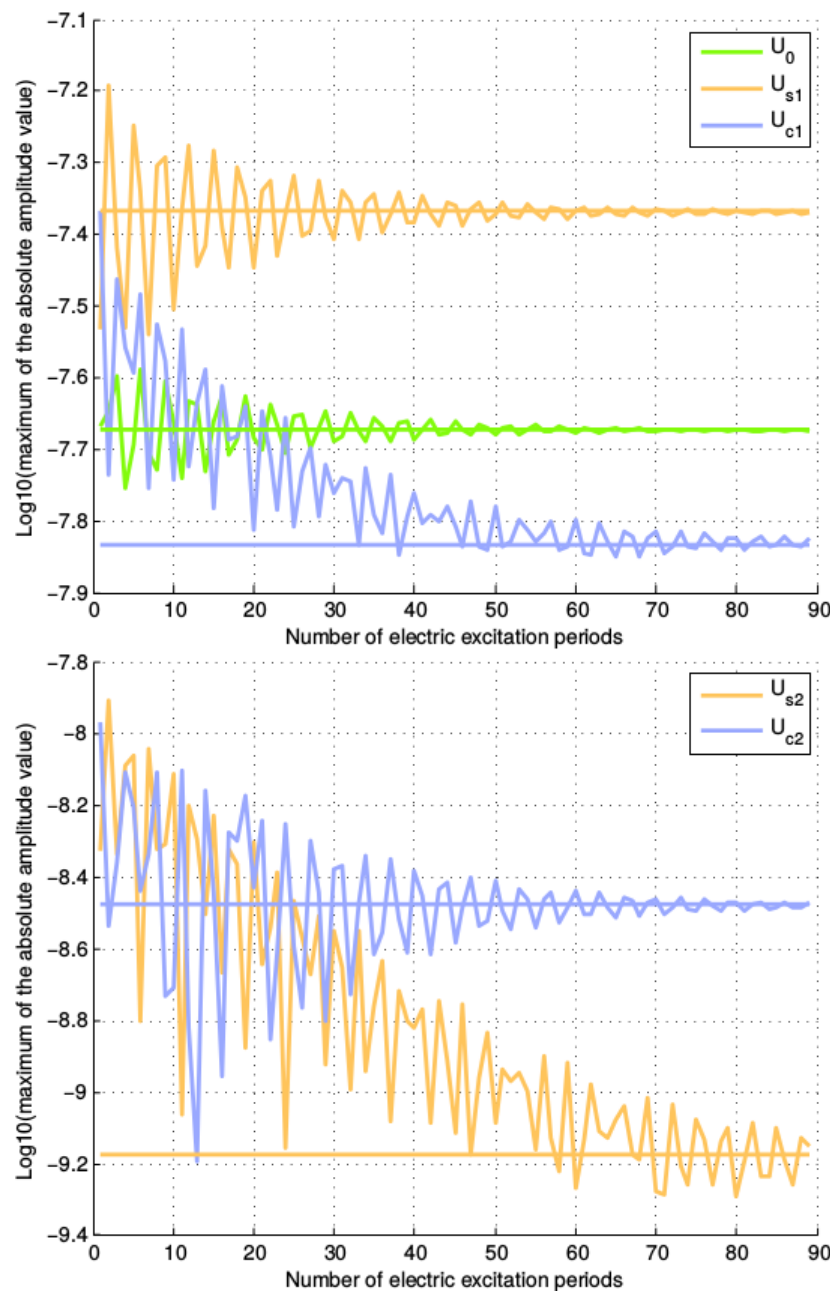

Fig. 9 Maximum of the multiharmonically computed displacement (m) harmonics (horizontal lines) and of the harmonics computed via a Fourier transform at every electric excitation period in the Newmark time resolution. Time increases in the right direction.

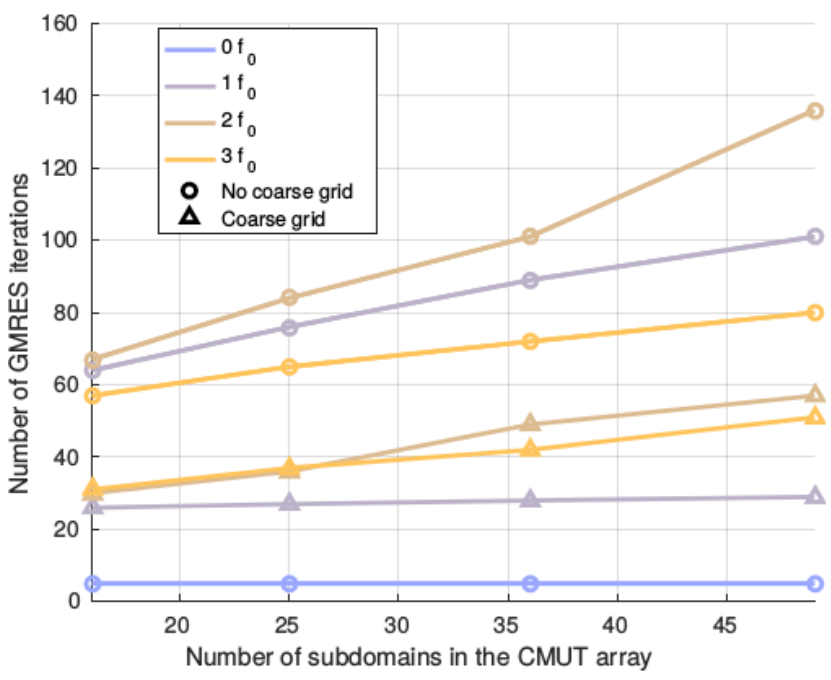

Fig. 10 Number of GMRES iterations to reach a $10^{-9}$ tolerance in the staggered elastoacoustic DDM resolution as the number of subdomains in the CMUT array increases. Results are shown for the 4 frequencies in the truncated Fourier series of the mechanical displacement and the acoustic pressure fields with and without the coarse grid preconditionner. Only the bottom left cell has a non-zero alternating electric excitation voltage added to the DC bias.

applied on all cells with an additional $70 \sin \left(2 \pi \cdot 10^{6} t\right)$ volts applied only on the bottom left cell and on the seventh cell of the upper row. Because the pull-in voltage is around 200 volts and the membrane vibrates close to resonance the displacements changed by up to $30 \%$ in the staggered nonlinear iterations and nonlinearity had thus to be taken into account. Without the coarse grid preconditionner, because of the size of the array, the DDM required a rather large amount of iterations to reach a low tolerance as visible on Fig. 10. The figure shows the number of iterations required to reach a $10^{-9}$ tolerance on the relative residual in the GMRES DDM solver of the elastoacoutic problem versus the size of the array for the 4 frequencies in the Fourier truncation of the mechanical displacement and acoustic pressure fields. The coarse grid preconditonner dramatically improves this aspect. It is worth noting how slow the increase is for the constant and the $1 f_{0}$ frequencies. This is closely related to the low crosstalk that has been observed on them. For the $2 f_{0}$ frequency displayed on Fig. 11 the crosstalk is the strongest and the iteration count still increases with the coarse grid but at a slower rate.

\section{Conclusion}

This paper has detailed a robust and efficient method to compute the nonlinear, steady-state behavior of large arrays of electrically actuated micromembranes vibrating in a fluid. The method combines a high-order multiharmonic finite element formulation with a staggered domain decomposition scheme, which was successfully applied to both $2 \mathrm{D}$ and $3 \mathrm{D}$ 

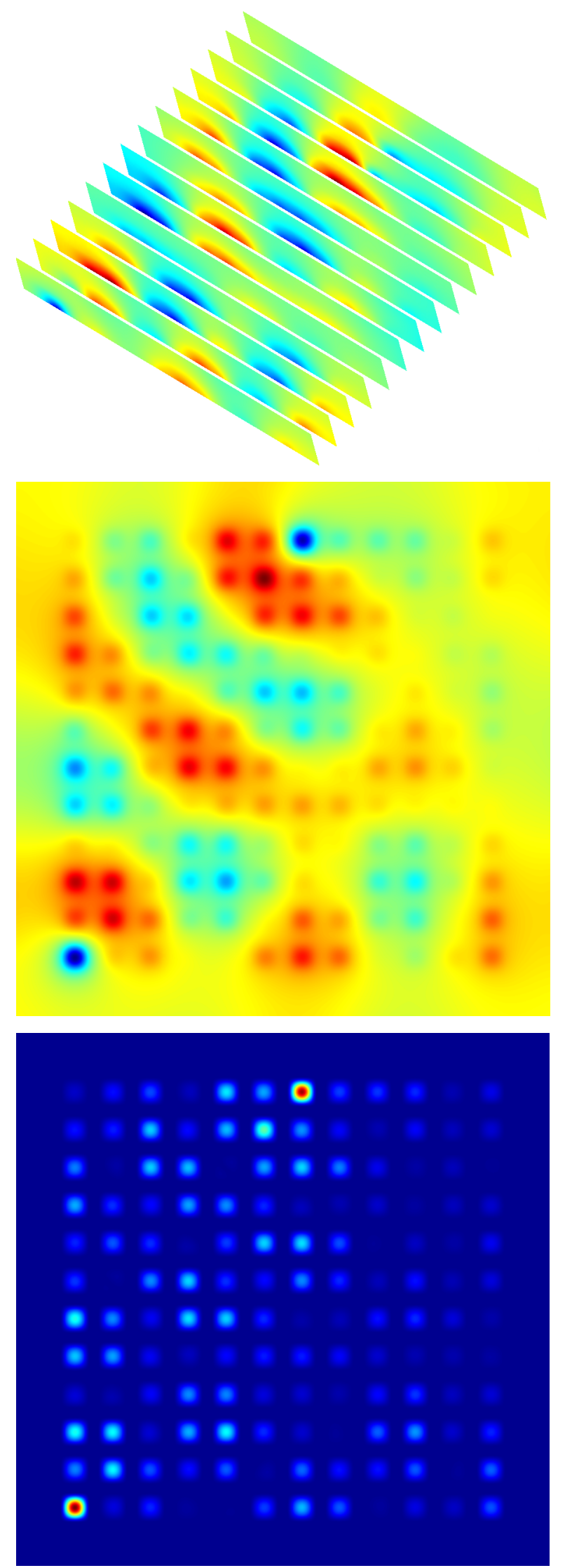

Fig. 11 Slices of the pressure harmonic $P_{c 2}$ versus vertical distance to the $12 x 12$ 3D CMUT array (top), pressure at the membrane top (middle) and mechanical displacement harmonic $\mathbf{U}_{c 2}$ (bottom). Positive pressures and displacements are in red, negative pressures in blue. Only the bottom left cell and the seventh cell of the upper row are excited with an alternating voltage. models of capacitive micromachined ultrasonic transducers (CMUTs). The speedup and robustness of the method has been demonstrated by comparing it to a classical time-stepping approach, and the complexity of the multiharmonic implementation has been shown to be dramatically reduced by using simple symbolic manipulations of the weak formulations. To illustrate the potential of this approach for realistic applications, a full 3D model of a CMUT with 144 membranes was simulated, where five harmonics were necessary to accurately capture the nonlinear, close-to resonant behavior of the system.

\section{Acknowledgment}

This work was supported in part by the Belgian Science Policy under grant IAP P7/02 and by an ARC grant for Concerted Research Actions (ARC WAVES 15/19-03), financed by the Wallonia-Brussels Federation. Computational resources have been provided by the Consortium des Equipements de Calcul Intensif (CECI), funded by the Fonds de la Recherche Scientifique de Belgique (F.R.S.-FNRS) under Grant No. 2.5020.11, and the Tier-1 supercomputer of the Federation Wallonie-Bruxelles, infrastructure funded by the Walloon Region under the grant agreement n1117545.

\section{References}

1. A. Caronti, G. Caliano, R. Carotenuto, A. Savoia, M. Pappalardo, E. Cianci, and V. Foglietti. Capacitive micromachined ultrasonic transducer (CMUT) arrays for medical imaging. Microelectronics Journal, 37(8):770-777, 2006.

2. J. Song, S. Jung, Y. Kim, K. Cho, B. Kim, S. Lee, J. Na, I. Yang, O.-L. Kwon, and D. Kim. Reconfigurable 2d CMUT-asic arrays for 3d ultrasound image. In SPIE Medical Imaging, pages 83201A-83201A. International Society for Optics and Photonics, 2012.

3. X. Wang, Y. Fan, W.-C. Tian, H.-J. Kwon, S. Kennerly, G. Claydon, and A. May. An air-coupled capacitive micromachined ultrasound transducer for noncontact nondestructive evaluation. In Sensors, 2007 IEEE, pages 1464-1467. IEEE, 2007.

4. C. O'Mahony, M. Hill, R. Duane, and A. Mathewson. Analysis of electromechanical boundary effects on the pull-in of micromachined fixed-fixed beams. Journal of Micromechanics and Microengineering, 13(4):S75, 2003.

5. S. Ballandras, M. Wilm, W. Daniau, A. Reinhardt, V. Laude, and R. Armati. Periodic finite element/boundary element modeling of capacitive micromachined ultrasonic transducers. Journal of applied physics, 97(3):034901, 2005.

6. M. Berthillier, P. Le Moal, and J. Lardies. Dynamic and acoustic modeling of capacitive micromachined ultrasonic transducers. In 2011 IEEE International Ultrasonics Symposium, pages 608-611. IEEE, 2011.

7. M. Berthillier, P. Le Moal, and J. Lardies. Comparison of various models to compute the vibro-acoustic response of large CMUT arrays. In Acoustics 2012, 2012.

8. N. Kacem, S. Baguet, S. Hentz, and R. Dufour. Computational and quasi-analytical models for non-linear vibrations of resonant MEMS and NEMS sensors. International Journal of Non-Linear Mechanics, 46(3):532-542, 2011. 
9. N. Kacem, A. Jallouli, V. Walter, G. Bourbon, P. Lemoal, and J. Lardies. Nonlinear dynamics of circular capacitive micromachined ultrasonic transducers. In SENSORS, 2015 IEEE, pages 1-4. IEEE, 2015.

10. A. Corigliano, M. Dossi, and S. Mariani. Domain decomposition and model order reduction methods applied to the simulation of multi-physics problems in MEMS. Computers \& Structures, 122:113-127, 2013.

11. N. M. Newmark. A method of computation for structural dynamics. Journal of the Engineering Mechanics Division, 85(3):67-94, 1959.

12. K-J. Bathe and E. L. Wilson. Numerical methods in finite element analysis. 1976.

13. J. Gyselinck, P. Dular, C. Geuzaine, and W. Legros. Harmonicbalance finite-element modeling of electromagnetic devices: a novel approach. Magnetics, IEEE Transactions on, 38(2):521$524,2002$.

14. B. Cochelin and C. Vergez. A high order purely frequency-based harmonic balance formulation for continuation of periodic solutions. Journal of sound and vibration, 324(1):243-262, 2009.

15. S. Karkar, B. Cochelin, and C. Vergez. A high-order, purely frequency based harmonic balance formulation for continuation of periodic solutions: The case of non-polynomial nonlinearities. Journal of Sound and Vibration, 332(4):968-977, 2013.

16. A. Halbach, P. Dular, and C. Geuzaine. Comparison of nonlinear domain decomposition schemes for coupled electromechanical problems. IEEE Transactions on Magnetics, In press, 2015.

17. D. M. Copeland and U. Langer. Domain decomposition solvers for nonlinear multiharmonic finite element equations. Journal of Numerical Mathematics, 18(3):157-175, 2010.

18. F. Bachinger, M. Kaltenbacher, and S. Reitzinger. An efficient solution strategy for the hbfe method. Proceedings of the IGTE, 2:385-389, 2002.

19. H. De Gersem, H. Vande Sande, and K. Hameyer. Strong coupled multi-harmonic finite element simulation package. COMPEL-The international journal for computation and mathematics in electrical and electronic engineering, 20(2):535-546, 2001.

20. G. Paoli, O. Biro, and G. Buchgraber. Complex representation in nonlinear time harmonic eddy current problems. Magnetics, IEEE Transactions on, 34(5):2625-2628, 1998.

21. S. Yamada and K. Bessho. Harmonic field calculation by the combination of finite element analysis and harmonic balance method. Magnetics, IEEE Transactions on, 24(6):2588-2590, 1988.

22. F. Bachinger, U. Langer, and J. Schöberl. Numerical analysis of nonlinear multiharmonic eddy current problems. Numerische Mathematik, 100(4):593-616, 2005.

23. F. Bachinger, U. Langer, and J. Schöberl. Efficient solvers for nonlinear time-periodic eddy current problems. Computing and Visualization in Science, 9(4):197-207, 2006.

24. A. Halbach and C. Geuzaine. Automatic derivation of multiharmonic formulations for nonlinear electromechanical problems with time dependent mesh deformation. In 2016 17th International Conference on Thermal, Mechanical and Multi-Physics Simulation and Experiments in Microelectronics and Microsystems (EuroSimE), pages 1-7. IEEE, 2016.

25. G. Li and NR. Aluru. A lagrangian approach for electrostatic analysis of deformable conductors. Microelectromechanical Systems, Journal of, 11(3):245-254, 2002.
26. S. Zaglmayr. High order finite element methods for electromagnetic field computation.

27. J. L. Coulomb. A methodology for the determination of global electromechanical quantities from a finite element analysis and its application to the evaluation of magnetic forces, torques and stiffness. IEEE Transactions on Magnetics, 19(6):2514-2519, 1983.

28. G. C. Everstine. Finite element formulations of structural acoustics problems. Computers \& Structures, 65(3):307-321, 1997.

29. V. Rochus, D. J. Rixen, and J.-C. Golinval. Monolithical modeling of electro-mechanical coupling in micro-structures. International Journal for Numerical Methods in Engineering, 65(4):474-482, 2006.

30. A. Halbach, P. Dular, and C. Geuzaine. Comparison of nonlinear domain decomposition schemes for coupled electromechanical problems. IEEE Transactions on Magnetics, 52(3):1-4, 2016.

31. H. A. Schwarz. Ueber einige abbildungsaufgaben. Journal für die reine und angewandte Mathematik, 70:105-120, 1869.

32. P.-L. Lions. On the Schwarz alternating method I.. In R. Glowinski, G. H. Golub, G. A. Meurant, and J. Périaux, editors, First International Symposium on Domain Decomposition Methods for Partial Differential Equations, pages 1-42. SIAM, Philadelphia, PA, 1988.

33. X.-C. Cai and M. Sarkis. A restricted additive Schwarz preconditioner for general sparse linear systems. SIAM Journal on Scientific Computing, 21(2):792-797, 1999.

34. C. Farhat and F.-X. Roux. A method of finite element tearing and interconnecting and its parallel solution algorithm. International Journal for Numerical Methods in Engineering, 32(6):1205-1227, 1991.

35. A. Vion and C. Geuzaine. Double sweep preconditioner for optimized Schwarz methods applied to the Helmholtz problem. $J$. Comput. Phys., 266:172-174, June 2014.

36. X. C. Cai, W. D Gropp, D. E Keyes, and M. D Tidriri. Newtonkrylov-schwarz methods in cfd. In Numerical methods for the Navier-Stokes equations, pages 17-30. Springer, 1994.

37. X.-C. Cai and D. E. Keyes. Nonlinearly preconditioned inexact newton algorithms. SIAM Journal on Scientific Computing, 24(1):183-200, 2002.

38. X. C. Cai. Nonlinear overlapping domain decomposition methods. In Domain Decomposition Methods in Science and Engineering XVIII, pages 217-224. Springer, 2009.

39. F. Confalonieri, A. Corigliano, M. Dossi, and M. Gornati. A domain decomposition technique applied to the solution of the coupled electro-mechanical problem. International Journal for $\mathrm{Nu}$ merical Methods in Engineering, 93(2):137-159, 2013.

40. T. F. Chan and T. P. Mathew. Domain decomposition algorithms. Acta numerica, 3:61-143, 1994.

41. Barry Smith, Petter Bjorstad, and William Gropp. Domain decomposition: parallel multilevel methods for elliptic partial differential equations. Cambridge university press, 2004.

42. R. Manwar and S. Chowdhury. Experimental analysis of bisbenzocyclobutene bonded capacitive micromachined ultrasonic transducers. Sensors, 16(7):959, 2016.

43. S. Marburg and B. Nolte. Computational acoustics of noise propagation in fluids: finite and boundary element methods, volume 578. Springer, 2008. 